\title{
Orthopaedic surgery in natural disaster and conflict settings: how can quality care be ensured?
}

\author{
Oscar Alvarado $^{1}$ - Miguel Trelles ${ }^{1,2}$ - Katie Tayler-Smith ${ }^{1,3} \cdot$ Holdine Joseph $^{1,4}$. \\ Rodné Gesline $^{1,4}$. Thélusma Eli Wilna ${ }^{1,4}$ - Mohammad Karim Mohammad Omar ${ }^{1,5}$. \\ Niaz Mohammad Faiz Mohammad ${ }^{1,5}$. John Muhima Mastaki ${ }^{1,6}$. \\ Richard Chingumwa Buhu ${ }^{1,6}$ • An Caluwaerts ${ }^{1,7}$. Lynette Dominguez ${ }^{1,2}$
}

Received: 26 February 2015 / Accepted: 29 March 2015 /Published online: 15 May 2015

(C) The Author(s) 2015. This article is published with open access at Springerlink.com

\begin{abstract}
Purpose Médecins sans Frontières (MSF) is one of the main providers of orthopaedic surgery in natural disaster and conflict settings and strictly imposes a minimum set of contextspecific standards before any surgery can be performed. Based on MSF's experience of performing orthopaedic surgery in a number of such settings, we describe: (a) whether it was possible to implement the minimum standards for one of the more rigorous orthopaedic procedures - internal fixation-and when possible, the time frame, (b) the volume and type of interventions performed and (c) the intra-operative mortality rates and postoperative infection rates.

Methods We conducted a retrospective review of routine programme data collected between 2007 and 2014 from three MSF emergency surgical interventions in Haiti (following the 2010 earthquake) and three ongoing MSF projects in Kunduz (Afghanistan), Masisi (Democratic Republic of the Congo) and Tabarre (Haiti).
\end{abstract}

Katie Tayler-Smith

katietaylersmith@gmail.com

1 Médecins sans Frontières - Operational Centre Brussels (MSF-OCB), Brussels, Belgium

2 MSF-OCB Surgery, Anaesthesia, Gynaecology, and Emergency Medicine Unit, Brussels, Belgium

3 MSF-OCB, Operational Research Unit, MSF-Luxembourg, Luxembourg, Luxembourg

4 Tabarre Referral Centre for Surgical and Orthopaedic Care, Port-au-Prince, Haiti

5 Kunduz Trauma Centre, Kunduz, Afghanistan

6 Masisi General Referral Hospital, North Kivu, Democratic Republic of the Congo

7 MSF-OCB Infection Control Unit, Brussels, Belgium
Results The minimum standards for internal fixation were achieved in one emergency intervention site in Haiti, and in Kunduz and Tabarre, taking up to 18 months to implement in Kunduz. All sites achieved the minimum standards to perform amputations, reductions and external fixations, with a total of 9,409 orthopaedic procedures performed during the study period. Intraoperative mortality rates ranged from 0.6 to $1.9 \%$ and postoperative infection rates from 2.4 to $3.5 \%$.

Conclusions In settings affected by natural disaster or conflict, a high volume and wide repertoire of orthopaedic surgical procedures can be performed with good outcomes when minimum standards are in place. More demanding procedures like internal fixation may not always be feasible.

Keywords Orthopaedic surgery · Minimum standards . Natural disaster and conflict settings $\cdot$ Internal fixation

\section{Introduction}

Physical trauma is a leading cause of global mortality, with natural disasters and armed conflicts contributing significantly $[1,2]$. As the latter most often occur in low- and middleincome countries (LMICs), it follows that these settings have the highest related trauma burden [3-6]. Many trauma cases require surgical intervention, particularly orthopaedic surgery. However, LMICs often lack the surgical capacity to meet these needs and thus there is a reliance on external actors.

Médecins sans Frontières (MSF), an international, independent, humanitarian medical organisation, provides medical assistance to vulnerable populations, including those affected by natural disasters and conflicts. Surgical care is an integral part of its work, and over the last 40 years, MSF has provided surgical care in natural disaster and conflict settings in more than 70 countries. Orthopaedic surgery (i.e. surgical treatment 
of injuries or disorders of the musculoskeletal system) constitutes a large part of this care.

To ensure quality surgical care, MSF has established a minimum set of context-specific standards that must be in place before any surgical procedures can be performed $[7$, 8]. This practice conforms to the Sphere guidelines on minimum standards in disaster response [9]. While such prerequisites can limit the possibilities of performing surgery, MSF has demonstrated that in many natural disaster and armed conflict settings it is possible to implement these minimum standards, even for more rigorous procedures like internal fixation.

The need for minimum standards to ensure delivery of quality care has been previously documented [10]. However, there are no studies to date reporting on whether it is possible to implement such standards for orthopaedic surgery (particularly more demanding procedures like internal fixation) in natural disaster and conflict settings and, when this is possible, the related outcomes.

Based on the MSF experience of performing orthopaedic surgical activities in natural disaster and conflict settings in Haiti, Afghanistan and the Democratic Republic of the Congo (DRC) we describe: (a) whether it has been possible to implement the minimum standards required for performing among the more rigorous orthopaedic procedures - internal fixation - and when possible, the approximate time frame, (b) the volume and type of orthopaedic activities performed and (c) the outcomes of these activities (intra-operative mortality rates and postoperative infection rates).

\section{Materials and methods}

\section{Study design}

This was a descriptive study using routine MSF programme data.

\section{Study setting}

The study was conducted in the surgical programmes of the MSF emergency intervention following the 2010 earthquake in Haiti (Cité Soleil, Chancerelles, Sarthe) and in three ongoing MSF projects in Kunduz (Afghanistan), Masisi (DRC) and Tabarre (Haiti). Specific details about the surgical capacity and available resources at each site are shown in Table 1.

\section{Cité Soleil, Chancerelles and Sarthe}

On 12 January 2010, Port-au-Prince was devastated by an earthquake. More than 200,000 people were killed and some 300,000 people injured [12]. At the time of the earthquake, MSF Operational Centre Brussels (OCB) had been operational in Haiti since 1991 and as such it was able to launch a rapid and well-organised response. Surgical activities were a primary focus and were established in two existing Ministry of Health $(\mathrm{MoH})$ structures (Cité Soleil and Chancerelles hospitals); meanwhile an old warehouse was converted into a hospital, and towards the end of February 2010 Sarthe hospital was opened exclusively for trauma care. All orthopaedic surgical activities were transferred to this facility.

\section{Masisi}

Masisi is an area of persistent conflict where MSF OCB, in collaboration with the $\mathrm{MoH}$, has been supporting a General Referral Hospital in North Kivu province since September 2007. In addition to the provision of free general health care, MSF also provides basic surgical/orthopaedic care at this hospital.

\section{Kunduz}

Afghanistan has suffered years of ongoing conflict, and MSF OCB fills a gap in the provision of trauma care through the Kunduz Trauma Centre which was opened in August 2011 in the northeastern province of Kunduz.

\section{Tabarre}

A Surgical and Trauma Centre was built new in the Tabarre neighbourhood of Port-au-Prince, Haiti and provides specialised care for visceral surgery and trauma patients, including orthopaedic care. It was created to address the gap in trauma care left by the 2010 earthquake. Quality standards were prioritised from the onset including infrastructure, material and human resources (qualified staff that had worked with MSF before in Sarthe hospital were hired). All types of trauma cases are seen at the centre.

\section{Orthopaedic procedures}

Orthopaedic interventions and their characteristics were classified according to a standardised system described previously [13]. At the time of the study, it was not possible to stabilise and treat any type of spinal fractures or provide prosthetic treatment for hip fractures, including open reduction and internal fixation.

\section{Minimum surgical standard requirements}

In MSF there are seven basic prerequisites for surgical care:

1. Adequate infrastructure, including protection from the external environment and appropriate electricity and lighting 


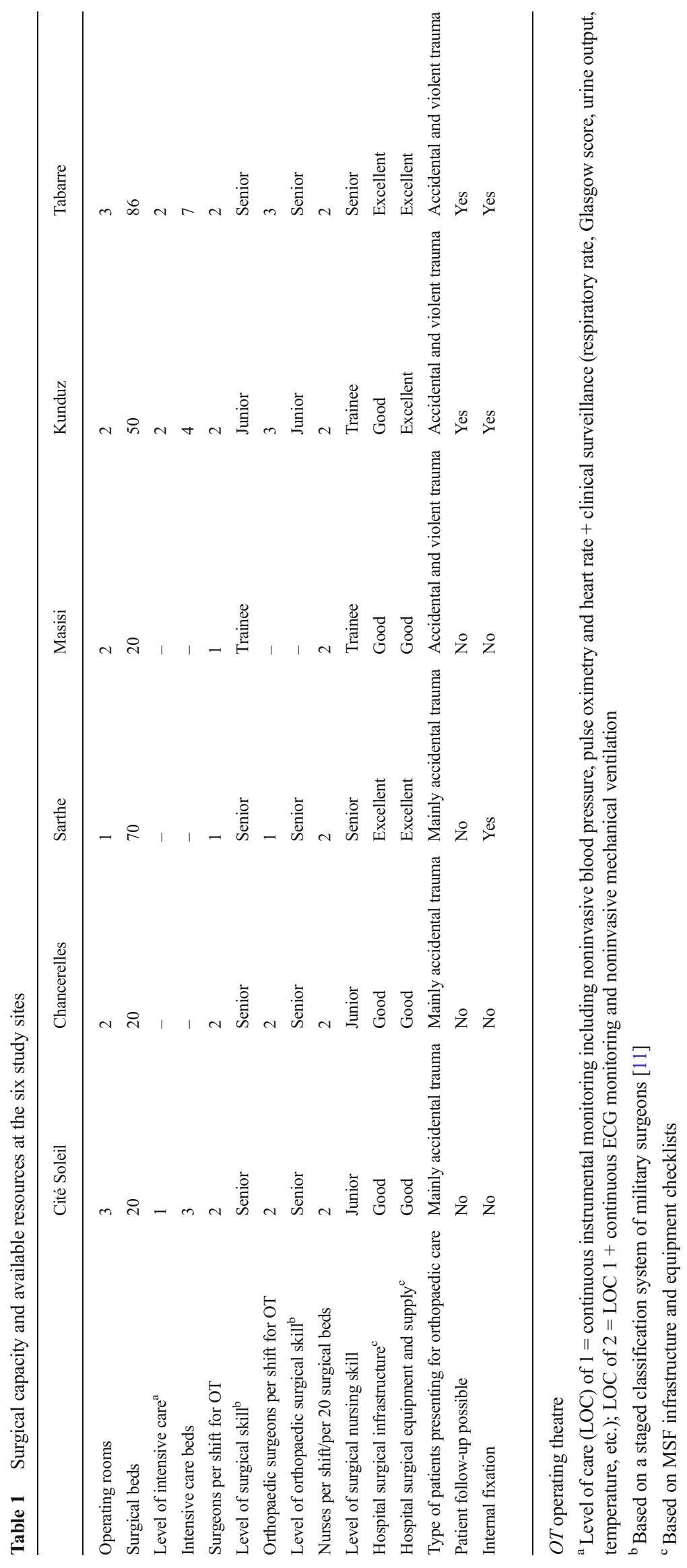


2. Adequate water and sanitation provisions, waste management being a key priority

3. Availability of all essential disposables, drugs and equipment

4. Strict adherence to hygiene requirements and universal precautions

5. Mandatory use of sterile equipment for surgical and anaesthesia procedures

6. Capability for blood transfusion

7. Adequate human resources in quantity and quality

These requirements are stepped up if internal fixation procedures are performed, due to the inherent risks and complications (e.g. osteomyelitis), including frequent assessment and supervision:

- Improved air quality with filters

- Availability of water supply in quantity and quality

- Special orthopaedic accessory table, C-arm and single-use gowns and drapes

- $\quad$ Reinforced dress code and flows, use of hydroalcoholic solutions and surface disinfectants

- Clearly defined clean and dirty circuits in sterilisation, autoclave (minimum 90 1) and instrument disinfectants

- Qualified orthopaedic surgeons, nurses and infection control officers, physiotherapy specialists

- Availability of antibiotic culture and sensitivity

\section{Inclusion/exclusion criteria}

Surgical interventions described in this study were limited to orthopaedic procedures and excluded any isolated soft tissue surgery not requiring specific orthopaedic instruments.

\section{Study period}

Data on the orthopaedic surgical activities performed at the different study sites for the following periods were included: Cité Soleil (16 January to 25 February 2010); Chancerelles (20 January to 25 February 2010); Sarthe (26 February 2010 to 6 June 2011); Masisi (3 September 2007 to 30 June 2014); Kunduz (30 August 2011 to 30 June 2014); and Tabarre (13 February 2012 to 30 June 2014).

\section{Data collection and analysis}

Data on all surgical/orthopaedic procedures performed in MSF programmes are routinely entered into a standardised logbook and transcribed into an electronic database (Microsoft Excel). Data for this study were sourced from this database. These data were aggregated at MSF OCB headquarters, reviewed for completeness and accuracy and validated by cross-checking with data held in individual patient records.

The data variables included: number of patients, number of surgical and orthopaedic procedures, type and number of orthopaedic procedures, intra-operative deaths and overall postoperative infection rate.

Intra-operative mortality was defined as any death occurring during the induction of anaesthesia, the intervention itself or the immediate recovery period. A postoperative infection was defined as any surgery-related infection identified while the patient was hospitalised or at the outpatient clinic.

Data were analysed in Microsoft Excel using basic summary statistics.

\section{Ethics}

The study satisfied the MSF Ethics Review Board (Geneva, Switzerland) criteria for studies using routinely collected data and was approved by the Comité National de Bioéthique of Haiti. The study was conducted as a retrospective analysis of routine programme data, and informed consent was thus not sought from study subjects; however, identifying information was removed from all patient records prior to analysis.

\section{Results}

\section{Cité Soleil and Chancerelles, Haiti}

While the basic minimum standards for surgery were able to be implemented, it was not possible to instigate the prerequisites needed for internal fixation in the short implementation period. Between 16 January and 25 February 2010, 170 orthopaedic procedures were performed. Table 2 shows the different types of procedures that were performed. Amputations and reductions made up the majority of procedures (53 and $33 \%$, respectively, in Cité Soleil and 18 and $75 \%$, respectively, in Chancerelles). The intra-operative mortality rate for all orthopaedic procedures was $1.9 \%$ in Cité Soleil and $0.6 \%$ in Chancerelles. Data on postoperative infections could not be captured due to the challenge of follow-up.

\section{Sarthe, Haiti}

After Sarthe hospital opened, it took 11 months to implement the prerequisites for performing internal fixation, with the first procedure being performed on 5 January 2011. Between 26 February 2010 and 6 June 2011, 731 orthopaedic procedures were performed, of which $6 \%$ were amputations, $25 \%$ reductions, $15 \%$ external fixations and $17 \%$ internal fixations. From the time when internal fixation began to be performed, it comprised $29 \%$ of the procedures (Table 2). There were no 
intra-operative deaths. Data on postoperative infections could not be captured.

\section{Masisi, DRC}

It was not possible to meet the prerequisites for internal fixation (namely adequate infrastructure and sterilisation, and qualified human resources); thus, it was not done. Between 3 September 2007 and 30 June 2014, 597 orthopaedic interventions were performed, of which $20 \%$ were amputations, $48 \%$ were reductions, $20 \%$ amputations and $7 \%$ external fixations (Table 2). The intra-operative mortality rate for all orthopaedic procedures was $0.2 \%$.

\section{Kunduz, Afghanistan}

From the time of the project launch, it took 18 months to put in place the prerequisites for internal fixation, and the first one was performed on 23 January 2013. Between 30 August 2011 and 30 June 2014, 4,026 orthopaedic procedures were performed, of which $5 \%$ were amputations, $41 \%$ reductions, $20 \%$ external fixations and $20 \%$ internal fixations. During 2013 , internal fixation procedures accounted for $32 \%$ of all procedures and in 2014 for $38 \%$ (Table 2). The intra-operative mortality rate for all orthopaedic procedures was $0.05 \%$. During 2013, the reported postoperative infection rate was $2.4 \%$.

\section{Tabarre, Haiti}

Built to cater for high level orthopaedic surgery, three days after opening, the first internal fixation procedure was performed, on 16 February 2012. Between 13 February 2012 and 30 June 2014, 4,119 orthopaedic procedures were performed, of which $2 \%$ were amputations, $16 \%$ reductions, $19 \%$ external fixations and $47 \%$ internal fixations. The proportion of internal fixations performed by year was consistent (Table 2). The intraoperative mortality rate for all orthopaedic procedures was $0.03 \%$. The postoperative infection rate was $2.8 \%$.

\section{Discussion}

This study demonstrates that in LMIC settings affected by natural disaster or conflict, a high volume and wide repertoire of orthopaedic surgical procedures can be performed with good outcomes when minimum standards are in place. More rigorous procedures like internal fixation may not always be feasible.

The high orthopaedic caseloads observed in this study emphasise the strong need for provision of orthopaedic surgery in settings afflicted by natural disaster and conflict. The challenge however is ensuring quality care against a backdrop of limited resources (material and human). Patients can end up 


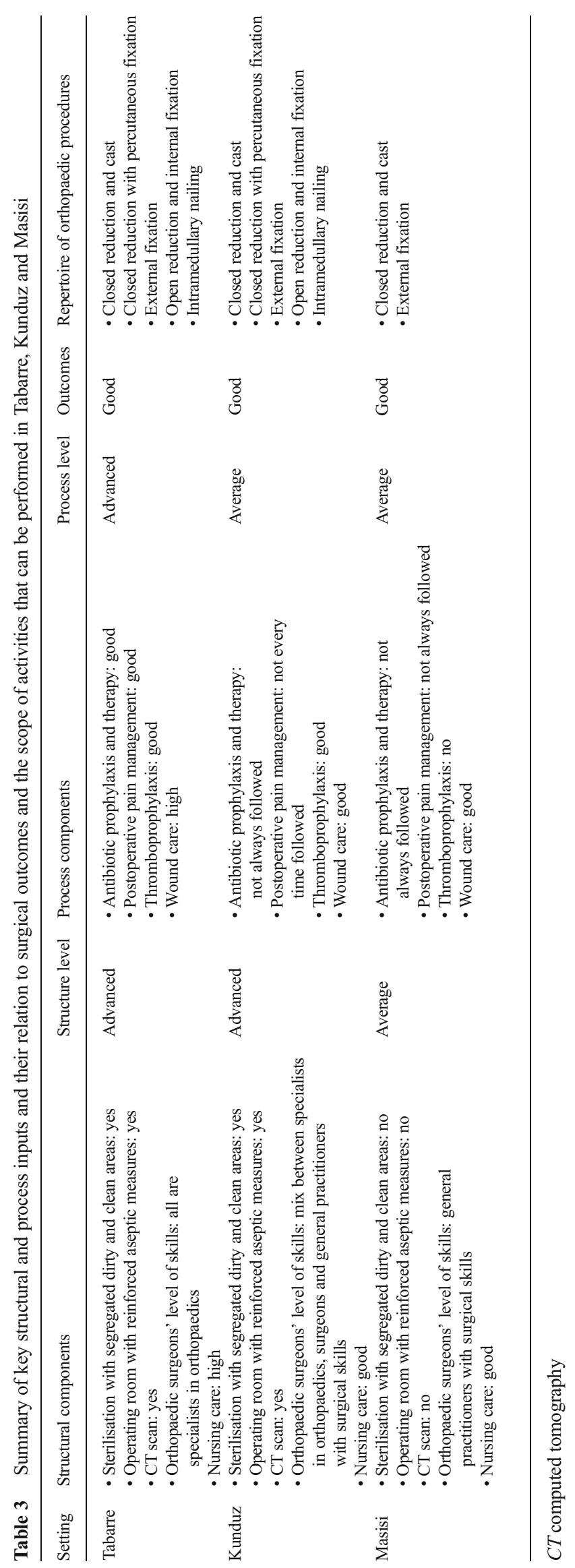


paying a high price for substandard surgical care, not least: postoperative infections (including osteomyelitis), disability and even death. The principle of "do no harm" should prevail at all times; if this principle cannot be upheld, then such surgery should not be undertaken at any cost.

According to the concepts outlined in the Donabedian model, quality of health care can be considered in the context of three domains: "structure", "process", and "outcomes" [14]. Structure describes the factors that affect the context in which care is delivered (e.g. hospital buildings, equipment, human resources); process defines the transactions between patients and providers during the delivery of health care (i.e. those factors that influence how health care is delivered); and outcomes refers to the effects of health care on the health status of patients and populations. The minimum standards of care endorsed by MSF for orthopaedic surgery ensure that all the necessary "structural" elements are in place for maximising quality of care. MSF ensures that important process factors are upheld through comprehensive and standardised protocols for all levels of care (universal precautions and hygiene, clinical therapeutic care: e.g. antibiotic prophylaxis, postoperative pain management, thromboprophylaxis, etc.), appropriate recordkeeping in patient files and anaesthesia and surgical records, and comprehensive data collection systems. In this study, we tried to assess the outcomes of these structural and process elements by looking at intra-operative mortality and postoperative infection rates. Low intra-operative mortality and postoperative infection rates across all study sites provide a fair indication that our quality of care is high. Table 3 summarises the interaction between structural and process inputs, outcomes and the scope of possible orthopaedic activities in three of our settings.

Basic orthopaedic procedures were possible in all of our study sites, while more demanding procedures such as internal fixation could only be performed at specific sites. Even then, the structural requirements needed to undertake this sort of procedure took as long as 18 months to implement in some settings. Staff expertise and skill level were large determining factors. Basic orthopaedic care can be performed by general surgeons, but more rigorous procedures, like internal fixation, unquestionably require the expertise of trained orthopaedic surgeons. And even when this level of expertise is available, the inherent risks of such a procedure need to be weighed up against the desired functional outcome for the patient; in some cases, conservative treatment of closed fractures may be more appropriate. That all said, we recognise that while internal fixation is among the more demanding procedures in terms of infrastructure and resource requirements, other types of surgical interventions might in fact be better indicated for many of the trauma cases and fractures that present, even when sophisticated operating theatres exist. In many cases, external fixation may be the more appropriate choice of intervention over internal fixation, especially in natural disaster and conflict settings. In this way, even in those settings where the minimum requirements for a procedure like internal fixation do not exist, a high proportion of the trauma caseload may be able to be managed when a procedure like external fixation is possible [15-19].

There were a number of study limitations. First, data on postoperative infection rates were not available at all the study sites. Nonetheless, given that the level of care we provide is standardised across our projects, we would not expect these rates to differ widely from those sites where these data were available. Second, the repertoire of outcome measures in our study was limited to intra-operative mortality and postoperative infection. Other useful indicators would have included infection rates after internal fixation (particularly osteomyelitis) and disability indicators. Complications after internal fixation have been assessed in other MSF trauma centres (namely MSF Operational Centre Paris in its orthopaedic programme following the 2010 earthquake in Haiti, where $5 \%$ of patients with internal fixation required this intervention to be repeated) [20]. Similar results might be expected in our study settings given that the policies, guidelines and protocols are the same.

In conclusion, in settings afflicted by natural disaster and conflict, where orthopaedic needs are high, good surgical outcomes can be achieved when context-specific minimum standards are in place. We make a strong call for providers of surgical health care in any setting, particularly LMIC settings, to ensure that minimum standards of care are in place before such surgery is undertaken and to avoid more rigorous procedures when these standards cannot be met.

Conflict of interest The authors declare that they have no conflict of interest.

Open Access This article is distributed under the terms of the Creative Commons Attribution 4.0 International License (http:// creativecommons.org/licenses/by/4.0/), which permits unrestricted use, distribution, and reproduction in any medium, provided you give appropriate credit to the original author(s) and the source, provide a link to the Creative Commons license, and indicate if changes were made.

\section{References}

1. World Health Organization (2010) Injuries and violence: the facts. World Health Organization, Geneva

2. Peden M, McGee K, Sharma G (2002) The injury chart book: a graphical overview of the global burden of injuries. World Health Organization, Geneva

3. Weiser TG, Regenbogen SE, Thompson KD, Haynes AB, Lipsitz SR, Berry WR, Gawande AA (2008) An estimation of the global volume of surgery: a modelling strategy based on available data. Lancet 372:139-144. doi:10.1016/S0140-6736(08)60878-8

4. Chu KM, Ford N, Trelles M (2010) Operative mortality in resourcelimited settings: the experience of Medecins Sans Frontieres in 13 countries. Arch Surg 145:721-725. doi:10.1001/archsurg.2010.137 
5. Nickerson JW, Chackungal S, Knowlton L, McQueen K, Burkle FM (2012) Surgical care during humanitarian crises: a systematic review of published surgical caseload data from foreign medical teams. Prehosp Disaster Med 27:184-189. doi:10.1017/ S1049023X12000556

6. Wong EG, Gupta S, Deckelbaum DL, Razek T, Kushner AL (2015) Prioritizing injury care: a review of trauma capacity in low and middle-income countries. J Surg Res 193:217-222. doi:10.1016/j. jss.2014.08.055

7. Timmermans S, Berg M (2003) The gold standard: the challenge of evidence-based medicine and standardization in health care. Temple University, Philadelphia

8. Pope C (2002) Contingency in everyday surgical work. Sociol Health Illn 24:369-384. doi:10.1111/1467-9566.00300

9. The Sphere Project (2004) Humanitarian charter and minimum standards in disaster response. 2004 edition. http://www. unrwausa.org/document.doc?id=7. Accessed 6 Feb 2015

10. World Health Organization (2012) Guide to infrastructure and supplies at various levels of health care facilities. World Health Organization, Geneva, www.who.int/surgery/publications/ s15983e.pdf. Accessed 6 Feb 2015

11. Hoencamp R, Tan E, Idenburg F, Ramasamy A, van Egmond T, Leenen L, Hamming J (2014) Challenges in the training of military surgeons: experiences from Dutch combat operations in southern Afghanistan. Eur J Trauma Emerg Surg 40:421-428. doi:10.1007/ s00068-014-0401-Z

12. Chu K, Stokes C, Trelles M, Ford N (2011) Improving effective surgical delivery in humanitarian disasters: lessons from Haiti. PLoS Med 8:e1001025. doi:10.1371/journal.pmed.1001025

13. Wong E, Trelles M, Dominguez L, Gupta S, Burnham G, Kushner AL (2014) Surgical skills needed for humanitarian missions in resource-limited settings: common operative procedures performed at Médecins sans Frontières facilities. Surgery 156:642-649. doi: 10.1016/j.surg.2014.02.002

14. Donabedian A (1966) Evaluating the quality of medical care. Milbank Mem Fund Q 44:166-203

15. Hinsenkamp M (2014) SICOT contribution to natural disaster assistance: the external fixator. Int Orthop 38:1549-1550. doi:10. 1007/s00264-014-2413-z

16. Boillot F, Herard P (2014) External fixators and sudden-onset disasters: Médecins Sans Frontières experience. Int Orthop 38:15511554. doi:10.1007/s00264-014-2344-8

17. Bertol MJ, Van den Bergh R, Trelles Centurion M, Kenslor Ralph DH, Basimuoneye Kahutsi JP, Qayeum Qasemy A, Jean J, Majuste A, Kubuya Hangi T, Safi S (2014) Saving life and limb: limb salvage using external fixation, a multicentre review of orthopaedic surgical activities in Médecins Sans Frontières. Int Orthop 38:1555-1561. doi:10.1007/ s00264-014-2451-6

18. Awais S, Saeed A, Ch A (2014) Use of external fixators for damage-control orthopaedics in natural disasters like the 2005 Pakistan earthquake. Int Orthop 38:1563-1568. doi:10.1007/ s00264-014-2436-5

19. Mathieu L, Ouattara N, Poichotte A, Saint-Macari E, Barbier O, Rongiéras F, Rigal S (2014) Temporary and definitive external fixation of war injuries: use of a French dedicated fixator. Int Orthop 38:1569-1576. doi:10.1007/s00264-0142305-2

20. Teicher CL, Alberti K, Porten K, Elder G, Baron E, Herard P (2014) Médecins sans frontières experience in orthopedic surgery in postearthquake Haiti in 2010. Prehosp Disaster Med 29:21-26. doi:10.1017/S1049023X13009278 